\title{
Cadenas agroindustriales y vocación productiva de ciudades intermedias de Uruguay: una aproximación por empleo
}

Agro-Industrial Chains and Productive Vocation of Intermediate Cities in Uruguay: An Employment Approach

Cadeias agroindustriais e vocação produtiva de cidades intermediárias do Uruguai: uma aproximação por emprego

María Noel Ackermann Barbero* Ángela Cortelezzi Ferreyra**

Recibido: 29 de noviembre de 2020

Aprobado: 13 de mayo de 2020

https://doi.org/10.12804/revistas.urosario.edu.co/territorios/a.8251

Para citar este artículo:

Ackermann Barbero, M. N., \& Cortelezzi Ferreyra, A. (2020). Cadenas agroindustriales y vocación productiva de ciudades intermedias de Uruguay: una aproximación por empleo. Territorios (43-Especial), 1-24. https://doi.org/10.12804/revistas.urosario.edu.co/territorios/a.8251

\begin{abstract}
* Oficina de Programación y de Politica Agropecuaria (OPYPA) del Ministerio de Ganadería, Agricultura $y$ Pesca (MGAP) de Uruguay. Correo electrónico: marianoelack@gmail.com. ORCID: https://orcid.org/ ORCID: 0000-0002-74382406

** Oficina de Programación y de Politica Agropecuaria (OPYPA) del Ministerio de Ganaderia, Agricultura y Pesca (MGAP) de Uruguay. Correo electrónico: angelacortelezzi@gmail. com. ORCID: https://orcid. org/0000-0002-8391-1496
\end{abstract}


Palabras clave

Especialización/ diversificación productiva; ciudades intermedias; cadenas agroindustriales.

Keywords

Productive specialization/diversification; intermediate cities; agro-industrial chains.

Palavras-chave

Especialização/ diversificação produtiva; cidades intermediárias; cadeias agroindustriais.

\section{territarias 43-Especial}

\section{RESUMEN}

Este estudio pretende identificar la vocación productiva (agro) de las ciudades intermedias de Uruguay (CIU), a partir del estudio de la especialización o diversificación del empleo generado para aportar a la comprensión de los procesos de desarrollo registrados en los territorios y las demandas de empleo asociadas. En particular, interesa encontrar patrones de diversificación o concentración de actividades económicas, a partir de datos de empleo en cinco cadenas productivas agroindustriales (CPA): forestal, soja, arroz, carne bovina y leche. Se elaboraron indicadores de especialización y diversificación por empleo, utilizando la Encuesta Continua de Hogares para las ciudades de más de 5000 habitantes de Uruguay, excluyendo Montevideo y su zona metropolitana. De las 44 localidades analizadas, 32 presentaron una especialización superior al promedio de la economía en las CPA mencionadas, lo que indica que las CIU dependen significativamente de estas actividades. Finalmente, las CIU se clasificaron en las siguientes categorías: con fuerte presencia de la administración pública y de servicios del Estado; con vocación productiva asociada al manejo intensivo de recursos naturales o a la transformación de materias primas e insumos; con especialización en la provisión de servicios transversales, y con dedicación a los servicios turísticos.

\section{Abstract}

This study aims to identify the productive configuration (agro) of Uruguayan intermediate cities (CIU, for its acronym in Spanish), studying the specialization and diversification of employment in order to understand the development processes registered in the territory and the employment demands associated. In particular, the objective is to find patterns of diversification or concentration of economic activities from employment data in five agro-industrial productive chains (CPA, for its acronym in Spanish): forestry, soybeans, rice, beef, and milk. Specialization and diversification indicators were constructed using the Continuous Households Survey of Uruguay, excluding Montevideo and its metropolitan area. Of the 44 cities analyzed, 32 presented a specialization coefficient above the economy average in the CPA mentioned, which indicates that the CIU depend significantly on these activities. Subsequently, cities were classified and identified in the following categories: cities with a strong presence of public administration activities and associated with the State services; others cities with a productive vocation associated with the intensive management of natural resources or the transformation of raw materials and inputs; a group of cities specialized in the provision of transversal services, and others with dedication to tourist services.

\section{RESUMO}

Este estudo pretende identificar a vocação produtiva (agro) das cidades intermediárias do Uruguai (CIU) a partir do estudo da especialização ou diversificação do emprego gerado para aportar à compreensão dos processos de desenvolvimento registrados nos territórios e as demandas de emprego associadas. Particularmente, interessa encontrar padres de diversificação ou concentração de atividades econômicas a partir de dados de emprego em cinco cadeias produtivas agroindustriais (CPA): florestal, soia, arroz, carne bovina e leite. Se elaboraram indicadores de 
especialização e diversificação por exemplo utilizando os Inquéritos Contínuos de Lares para as cidades de mais de $\mathbf{5 0 0 0}$ habitantes do Uruguai, excluindo Montevideo e sua zona metropolitana. Das 44 localidades analisadas, 32 apresentaram uma especialização superior à média da economia nas CPA mencionadas, o que indica que as cidades intermediárias do Uruguai dependem significativamente destas atividades. Finalmente, as cidades intermediárias se classificaram nas seguintes categorias: com forte presença da administração pública e de serviços do Estado, com vocação produtiva associada ao manejo intensivo de recursos naturais ou à transformação de matérias primas e insumos, com especialização na provisão de serviços transversais e com dedicação nos serviços turísticos.

\section{Mecanismos de organización territorial de la producción: algunas consideraciones teóricas}

La literatura sobre la localización de las actividades económicas muestra que las empresas no se localizan uniformemente en el territorio, sino que tienden a concentrarse en áreas determinadas aprovechando la dotación de recursos naturales y las economías de escala y de alcance que surjan de la aglomeración de empresas y personas, entre otros factores, que permiten potenciar la productividad y competitividad, y configurar patrones de especialización regional. Goinheix et al. (2014) sostienen, con base en Vázquez Barquero (2005), que hay cuatro fuerzas que explican "en interacción sinérgica" los procesos de desarrollo de un territorio: la especialización y organización productiva en el territorio; el capital humano y el conocimiento; las economías de aglomeración urbana, y el entramado social y urbano.

La literatura de teoría económica de redes y sistemas define diferentes categorías de organización productiva del territorio: cadena productiva/cadena de valor, clústeres, sistemas productivos locales o distritos productivos y redes de empresas (Goinheix et al., 2014). En particular, los clústeres y los sistemas productivos locales o distritos productivos pueden presentar un anclaje en el territorio a nivel de ciudades, regiones o localidades.

Los clústeres se definen como grupos de empresas e instituciones enlazadas en un sistema de valor, con determinada concentración geográfica de actividades, que permiten especialización. La coordinación de todas las actividades entrelazadas reduce los costos de transacción, donde la gestión y la articulación de todo ese sistema son la fuente clave de ventajas competitivas. Los clústeres ponen énfasis en la competitividad sistémica apoyada en la especialización geográfica, sin involucrar necesariamente dentro de sus objetivos y funcionamiento la idea de un proyecto de desarrollo integral para el territorio (Goinheix et al., 2014).

La organización en sistemas productivos locales se caracteriza por la especialización productiva en un territorio, territorios 43-Especial 


\section{territorias} 43-Especial en el que se desarrolla la actividad - en general se especializan en un producto con diversos tipos de complejidad y uso final- de manera descentralizada, a través de unidades que mayormente son de pequeña o mediana dimensión. Son entidades socioterritoriales en las que el desarrollo social y económico del territorio no puede disociarse. Se generan redes a través de la cooperación formal e informal entre empresas y con otros actores locales como gobierno, asociaciones empresariales, instituciones de créditos y centros de investigación (Goinheix et al., 2014; Naclario et al., 2010).

Goinheix et al. (2014) señalan entre las ventajas de los sistemas productivos locales: disponibilidad de masa crítica de trabajadores calificados y especializados - que reduce los costos de búsqueda y reclutamiento de personal-; facilidad para conseguir e intercambiar información comercial y técnica entre empresas; intercambio formal e informal; aprendizaje y educación mutua, y generación de un ambiente propicio para la creación, adaptación y difusión de innovaciones en un proceso productivo ligado a la vida social de las personas, que comprende más que el ámbito puramente técnico. Asimismo, Naclario et al. (2010) señalan que existen ámbitos de cooperación entre las empresas de un mismo sector para dar respuestas colectivas a problemas del conjunto: necesidad de incrementar existencias para la exportación o satisfacer nichos internos de mercado, necesidad de convertirse en referentes zonales de un tipo de producto determinado, abaratamiento de costos - compras conjuntas, fletes, etc.—, diseño de estrategias de mercadeo o de sistemas electrónicos de salida al mercado.

Para que una ciudad o localidad pueda identificarse como distrito o sistema productivo local no debe haber empresas (una o pocas) que dominen la producción; por el contrario, debe coexistir un conjunto de empresas, en general de tamaño mediano o pequeño, que se concentren en ciertas actividades y fases productivas - actividad principal, rodeada de sectores auxiliares de apoyo a esta- (Climent, 1997; Naclario et al., 2010).

Las economías de aglomeración urbana son un factor relevante para explicar la diversificación de las ciudades. En estas importa considerar tres factores: las economías de escala y costos de transporte, el tamaño de los mercados y las economías de urbanización (Goinheix et al., 2014). Por lo tanto, dentro de los factores que se asocian a la diversificación productiva a nivel regional se señala la distancia respecto a los grandes mercados, que dan lugar a que las regiones periféricas tengan estructuras productivas menos diversificadas. El grado de diversificación está también relacionado positivamente con el tamaño de la población o densidad poblacional, según estudios empíricos. La radicación de empresas en zonas densamente pobladas se debe a los altos costos de transporte que deben enfrentar ciertos tipos de industrias (Jaramillo, 2016). Entre los beneficios 
asociados a la diversificación productiva regional se indican los efectos derrame por la interacción de firmas de diferentes sectores; incremento de actores que conforman la matriz productiva y de las relaciones entre ellos, y una menor exposición a shocks externos (Jaramillo, 2016).

\section{Metodología}

\section{Consideraciones metodológicas generales y fuente de datos}

Se calcularon indicadores de especialización y diversificación por empleo a partir de la Encuesta Continua de Hogares (ECH) del Instituto Nacional de Estadísticas (INE) para las ciudades de más de 5000 habitantes, entre 2013 y 2017. La ECH permite obtener información sometida a crítica, validada y específica tanto del empleo como de las características socioeconómicas de las personas desagregadas para las entidades territoriales definidas. Desde el año 2006, la ECH cuenta con una cobertura total del país.

Como toda encuesta, la ECH tiene limitantes: se construye a partir de muestras de la población objetivo (con los errores asociados a las técnicas de muestreo) y su precisión puede ser cuestionada en pequeñas poblaciones (su representatividad territorial anual permite, en principio, la desagregación departamental). Sin embargo, su principal ventaja es que se diseña para relevar variables socioeconómicas y, en particular, las asociadas a la condición de ocupación de los individuos (empleo formal e informal), lo cual no está presente en otras fuentes de datos.

El tamaño de la muestra puede resultar una limitante cuando se realizan estimaciones con gran nivel de desagregación, por lo que se generó una nueva base agrupando 5 años de encuestas (2013 a 2017). Esto determinó un tamaño de muestra de 618102 observaciones. La estrategia adoptada con el objetivo de generar estimaciones a nivel de ciudades de más de 5000 habitantes surge de la revisión de distintos antecedentes bibliográficos existentes en Uruguay (Goyeneche et al., 2011; Rodríguez Miranda, 2011; 2013). Para determinar si el tamaño muestral obtenido en cada ciudad y por grandes categorías de actividad (que se definirán más adelante) es suficiente desde el punto de vista estadístico para generar estimaciones se utilizó como criterio que las observaciones relevadas fueran mayores o iguales a $30 .^{1}$

Para localizar el empleo asociado a las cadenas agroindustriales bajo análisis se empleó el Clasificador Industrial Internacional Uniforme en su cuarta revisión (CIIU revisión 4), con desagregación a 4 dígitos. A partir de las preguntas que relevan el sector de actividad en que está empleado, se clasificó a los trabajadores en las distintas cadenas en estudio y sus fases. Para esto se consideró la ocupación principal, definida como aquella que reporta mayores ingresos.
1 Este estudio pretende caracterizar la especialización de las ciudades por empleo en términos de proporciones, las cuales se asimilan a una media muestral cuando el experimento aleatorio realizado es de carácter binomial. En este sentido, el Teorema Central del Limite establece que para muestras aleatorias grandes la distribución de probabilidad para las medias muestrales se aproxima a una distribución normal. Esto implica que se puede aproximar la distribución de las medias muestrales sin contar con información del comportamiento o distribución original de la población de la cual se toma la muestra. Si la población está normalmente distribuida, para cualquier tamaño de muestra se deduce que la distribución de la media muestral también será normal. Si la población no es normal o se desconoce su distribución, como sucede en este caso, una regla empirica habitualmente utilizada por los estadisticos indica que una muestra de tamaño 300 superior es suficiente para aplicar el Teorema Central del Limite, aproximar

territarios 43-Especial 
$\Leftarrow$

la distribución del estadistico utilizado y estimar intervalos de confianza para las estimaciones puntuales utilizando la distribución normal.

${ }^{2}$ http://www. fadu.edu. uy/itu/ciu/

\section{territarios 43-Especial}

De esta forma, se obtuvo una estimación de los puestos de trabajo generados en cada cadena y fase. La estimación obtenida constituye un mínimo para los puestos de trabajo asociados, ya que podrían existir individuos que estén ocupados en más de un puesto a la vez. De todas maneras, la incidencia del trabajo secundario en el sector agroindustrial es baja, por lo que las proporciones obtenidas a partir de la ocupación principal no se verían afectadas sustancialmente y no cambiarían las conclusiones.

Las CPA analizadas son las de la soja, la forestación, el arroz, la carne y la leche. Cada cadena se dividió en dos fases: producción primaria e industrialización. Además, se estimó en forma agregada el empleo asociado a los servicios de apoyo a la producción en la fase primaria agropecuaria; a las actividades de comercialización mayorista de productos y provisión de insumos y bienes de capital para la producción, y a la logística (transporte y manipulación de cargas, almacenamiento). Este último agregado se denominó "Actividades transversales".

En el Anexo 1 se detallan las correspondencias entre los códigos CIIU revisión 4 y las cadenas/fases definidas en las CPA, así como algunas consideraciones para tener en cuenta respecto a las estimaciones obtenidas de acuerdo con la definición de las distintas actividades en el clasificador.

\section{Metodología de identificación de las ciudades intermedias de Uruguay (CIU)}

Las CIU a analizar (localidades de más de 5000 habitantes sin incluir Montevideo y sus zonas metropolitanas) se determinaron considerando dos aspectos esenciales:

- La población relevada en el último Censo General de Población llevado a cabo por el Instituto Nacional de Estadísticas (INE) en el año 2011.

- Reagrupamiento de localidades listadas por el INE, que funcional y operativamente constituyen un mismo centro poblado con aquellas ocupaciones urbanas del suelo comprendidas en un radio de 6 kilómetros de la centralidad principal y que no forman parte de la conurbación metropolitana, lo cual fue realizado por el Instituto de Teoría y Urbanismo de la Facultad de Arquitectura, Diseño y Urbanismo ${ }^{2}$ de la Universidad de la República (Martínez et al., 2013).

Bajo estos criterios se definieron 45 conglomerados de más de 5000 habitantes por fuera del área metropolitana. Para evitar problemas de insuficiencia de muestra al efectuar las estimaciones, se decidió considerar las localidades de Pan de Azúcar y Piriápolis de manera conjunta. De este modo, quedaron conformados un total de 44 conglomerados urbanos. 
Indicadores de la diversificación productiva territorial a través del empleo

Para medir el grado de concentración en una industria o mercado se utilizó el Índice de Herfindahl-Hirschman ( $\mathrm{IHH}$, definido como la sumatoria de la participación de cada sector de la variable medida (en este caso el empleo) en el total regional, elevada al cuadrado. El inverso del IHH ( ID $\left._{\mathrm{IHH}}\right)$ constituye un indicador del grado de diversificación regional, de manera que el valor del índice se incrementa a medida que aumenta la diversidad regional (Jaramillo, 2016; Rodríguez Miranda, 2013).

$$
\begin{gathered}
I H H_{i}=\sum_{j=1}^{J}\left(\frac{\text { ocupados }_{i, j}}{\text { ocupados }_{i}}\right)^{2} \\
I D_{I H H_{i}}=\frac{1}{I H H_{i}}
\end{gathered}
$$

Otro indicador utilizado para medir la dispersión es el Coeficiente de Entropía $\left(I E_{j}\right)$, donde valores mayores corresponden a regiones más diversificadas.

$$
I E_{i}=\sum_{j=1}^{J}\left(\frac{\text { ocupados }_{i, j}}{\text { ocupados }_{i}}\right) * \log \left(\frac{1}{\left(\frac{\text { ocupados }_{i, j}}{\text { ocupados }_{i}}\right)}\right)
$$

Donde, en ambos indicadores:

$i$ : ciudad intermedia de Uruguay

$j$ : sector de actividad
Si todos los ocupados se encontraran en un único sector, el grado de dispersión sería mínimo, alcanzando el coeficiente su valor mínimo ( $I E_{j}$ valdría cero), mientras que si todos los sectores presentaran igual nivel de ocupación en una región (todos poseen la misma cuota de mercado), la entropía sería máxima y su valor sería igual al logaritmo del número total de sectores. En consecuencia, el valor del índice de entropía está acotado entre 0 y el logaritmo del número de sectores. Cabe señalar que cuando la participación en el empleo en un sector de actividad es nula no puede definirse el valor del índice. ${ }^{3}$

\section{Indicador de la especialización productiva territorial a través del empleo}

Para medir la especialización en las ciudades de más de 5000 habitantes se definió y calculó el Coeficiente de Especialización productiva aproximado por Empleo (CEE), por sector de actividad económica (Rodríguez Miranda, 2013):

$$
C E E=\frac{\left(\frac{\sum \text { ocupados }_{i, j}}{\sum_{\text {ocupados }}}\right)}{\left(\frac{\sum \text { ocupados }_{j}}{\sum \text { ocupados }_{\text {pais }}}\right)}
$$

Donde:

$i$ : ciudad intermedia de Uruguay

$j$ : sector de actividad
${ }^{3}$ Para el cálculo del índice de entropia, se reemplazaron los casos en los que no hay ocupados en algunas ramas de algunas regiones por 1, para evitar que el coeficiente quedara indefinido. 


\section{territorias} 43-Especial 8
Este indicador puede utilizarse para interpretar la especialización productiva relativa de una localidad en términos de empleo. Un CEE mayor a uno indica que la ciudad intermedia $i$ presenta una especialización relativa superior en el sector de actividad $j$ en comparación con el promedio nacional; mayor será dicha especialización cuanto más alto sea este indicador. Lo contrario se deduce si el valor del CEE es menor a uno para una ciudad intermedia $i$ en un sector de actividad $j$. De este modo, para identificar un potencial efecto de especialización aproximado por empleo en las CPA bajo estudio se considerarán aquellas CIU que presenten un CEE superior a la unidad en estas actividades.

Asimismo, se agruparon los sectores de actividad de la economía para mejorar la lectura y obtener mayor claridad sobre la especialización, en particular, en lo referente a las CPA bajo estudio y los servicios asociados. Como alternativa, para conservar la suficiencia de muestra, se realizó el análisis por fase dentro de cada CPA: primaria, industrial y actividades transversales.

A su vez se definieron seis agregados para el resto de las actividades económicas:

- Actividades agroindustriales no CPA: comprende las actividades primarias y agroindustriales que no forman parte de los rubros de estudio.

- Industrias: comprende la industria manufacturera no vinculada a las CPA y el sector de construcción.
- Servicios de distribución y comercialización: actividad comercial, transporte y almacenamiento (se excluye el sector logístico considerado dentro de las actividades trasversales en la CPA).

- Servicios de esparcimiento: alojamiento y servicios de comidas, arte y recreación.

- Servicios a la población: administración pública, enseñanza, salud y servicios sociales.

- Servicios de información, financieros y otros: informática y comunicaciones, actividades financieras y de seguros; servicios profesionales, técnicos e inmobiliarios; actividades administrativas y servicios de apoyo, y otras actividades de servicios y otros sectores que no queden comprendidos en los rubros anteriores.

\section{Resultados obtenidos de los indicadores de especialización/ diversificación}

A nivel general, Colonia del Sacramento, Canelones, Fray Bentos, Minas, Paysandú, Salto, San José de Mayo, Carmelo y Santa Lucía se encuentran entre las CIU con mayor diversificación productiva en términos relativos. Por otro lado, Lascano, Tarariras, Tranqueras y José Pedro Varela son las ciudades con menor diversificación de actividades económicas tanto por el IHH como por el indicador de entropía y reflejan, por lo tanto, economías más especializadas (figuras 1 y 2 ). 
Figura 1. Índice de diversificación productiva de Herfindahl

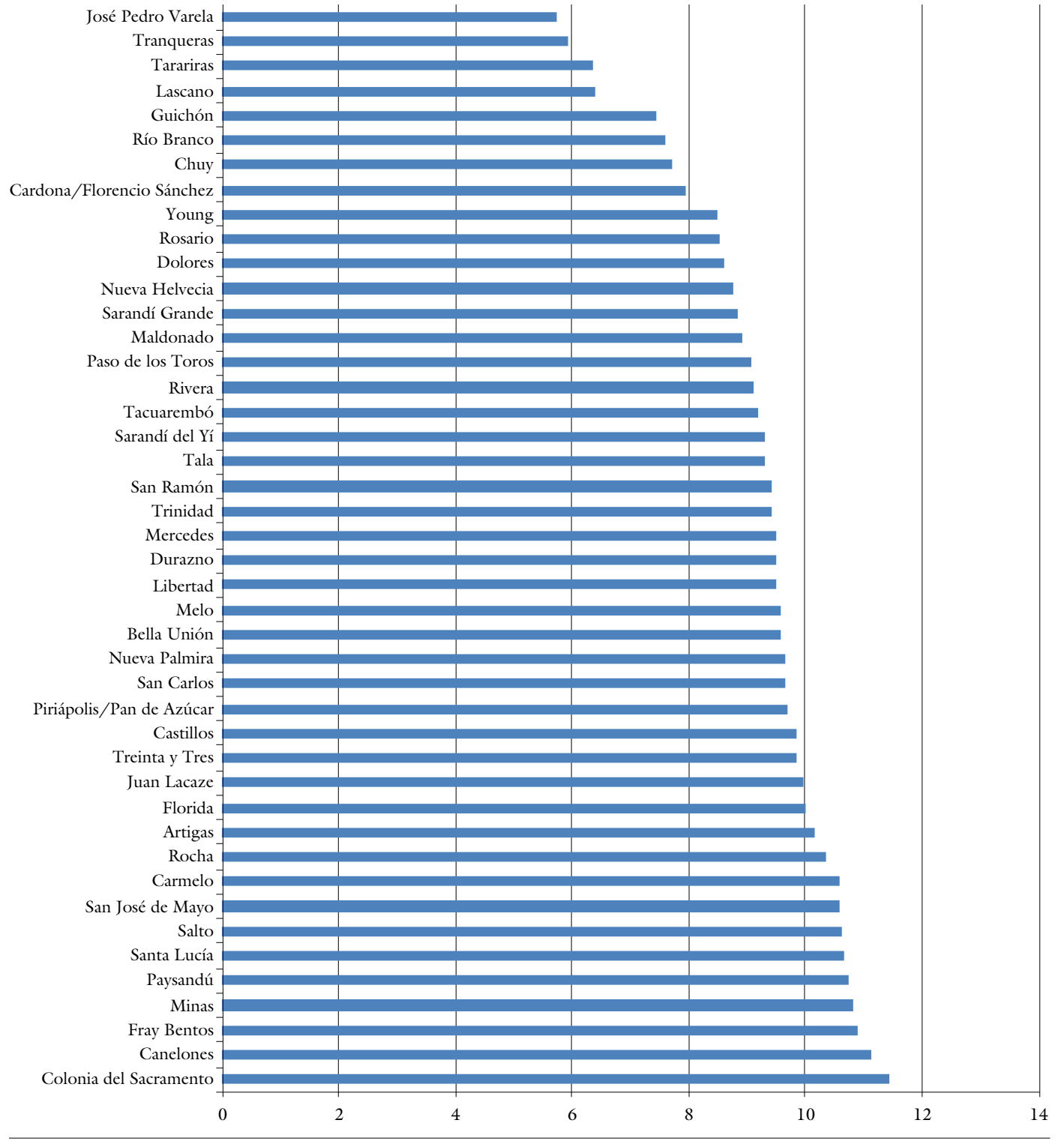

Fuente: elaboración propia con base en las ECH 2013 al 2017 del INE.

CADENAS AGROINDUSTRIALES Y VOCACIÓN PRODUCTIVA DE CIUDADES INTERMEDIAS DE URUGUAY

\section{territarios} 43-Especial 
Figura 2. Índice de diversificación productiva de entropía

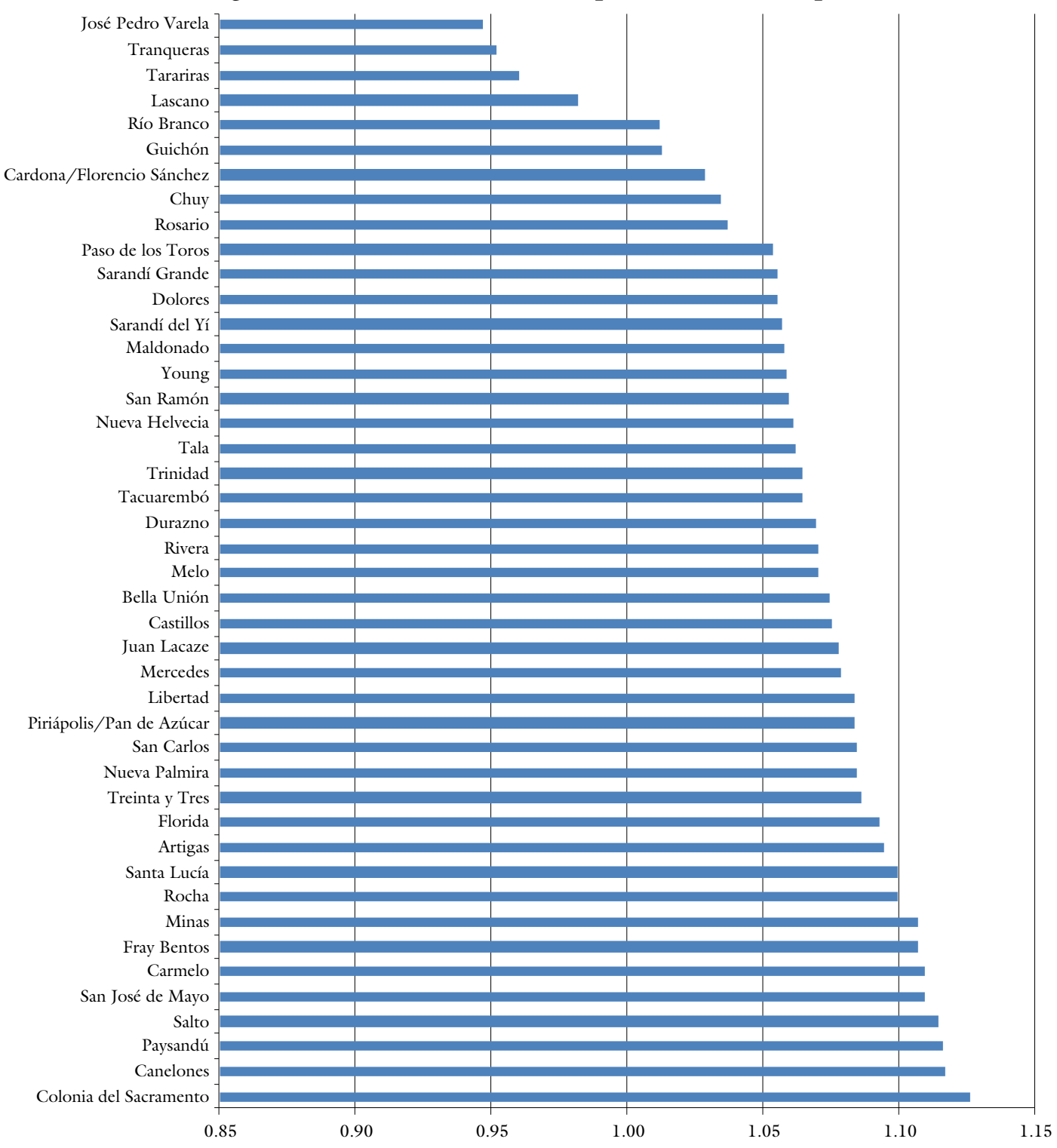

\section{tersitarias} 43-Especial

Fuente: elaboración propia con base en ECH 2013 al 2017 del INE. 
Con relación al CEE, que permite además observar los sectores de especialización de las ciudades, se observa que 32 de las 44 CIU analizadas registran un CEE mayor o igual a uno en el conjunto de las CPA, lo que indica una especialización productiva igual o superior a la observada para el promedio nacional en alguna de las CPA definidas (figura 3). Específicamente, 24 de las 44 localidades intermedias analizadas registran un CEE máximo y superior a la unidad en las CPA estudiadas.
El Anexo 2 presenta el listado de ciudades consideradas, el tamaño de muestra logrado con la estrategia adoptada, la estimación puntual de la proporción de empleo en las CPA en el total de la localidad (que corresponde al numerador del CEE) y su intervalo de confianza al $95 \%$.

Al analizar los perfiles de especialización productiva de cada una de las ciudades intermedias se observan algunos patrones (ver figura 4). Las localidades con mayor especialización productiva relativa en las CPA respecto al país son José Pedro

Figura 3. Coeficientes de especialización productiva aproximados por empleo para CPA por CIU

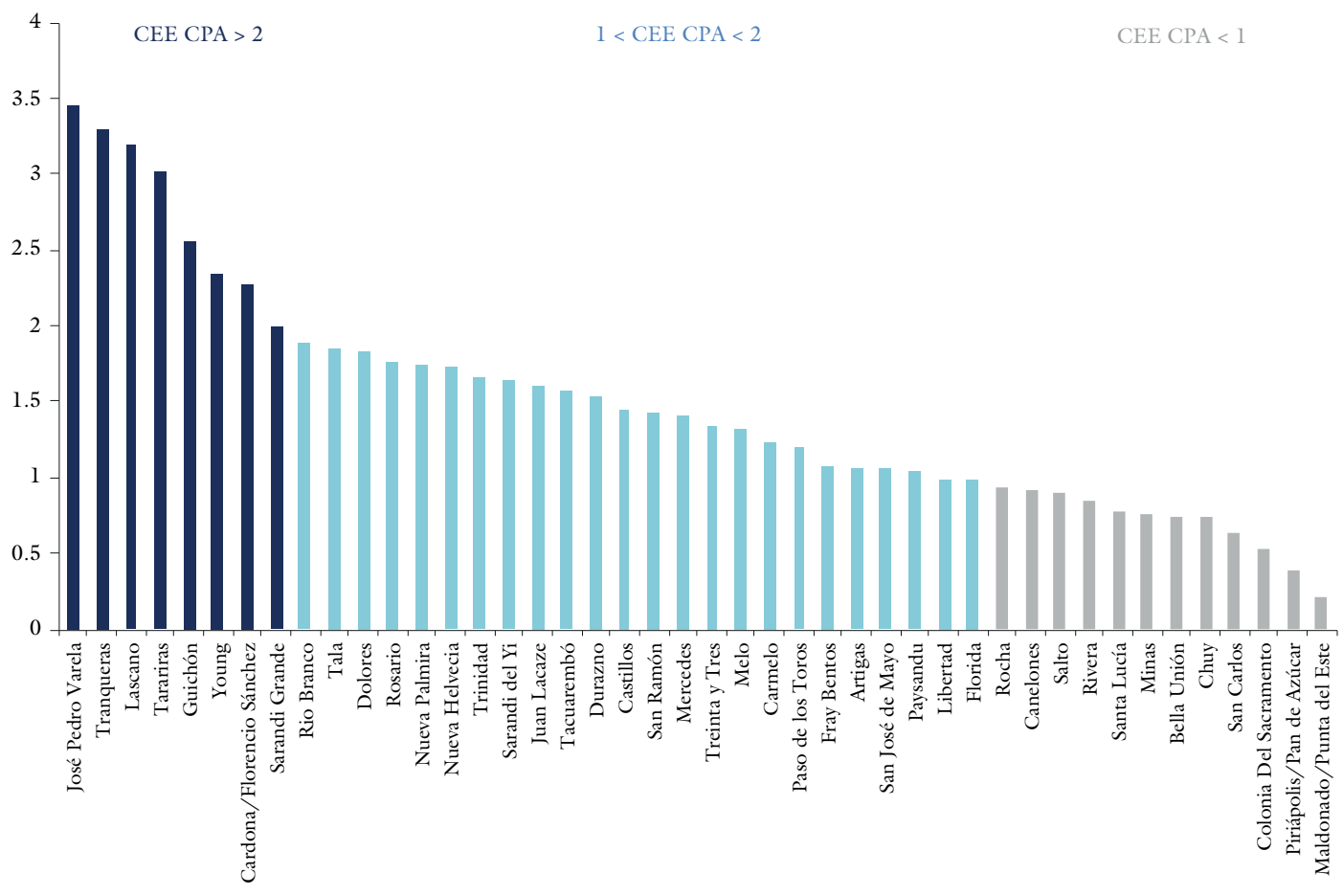

Fuente: elaboración propia con base en ECH 2013 al 2017 del INE. 
Varela y Tranqueras. La primera registra una mayor especialización en el cultivo de arroz y su procesamiento, la etapa primaria de la ganadería y servicios en predio. Tranqueras se caracteriza por la predominancia de sistemas silvo-pastoriles (mayor importancia relativa de la producción forestal, ganadera, servicios en predio), así como de la industria forestal, junto con otras actividades primarias no incluidas en las CPA analizadas (horticultura).

Guichón y Tacuarembó presentan perfiles similares en cuanto a la preponderancia del sector forestal. En Tacuarembó sobresale también la especialización en la industria cárnica, el sector arrocero y en los servicios en predio y logística. En tanto, en Guichón también se registra una fuerte presencia de ocupados en el área sojera y en servicios en predio.

Tarariras, Tala y Rosario presentan un perfil asociado a la ganadería de carne vacuna, leche y soja. En los casos de Tarariras y Rosario destacan además la mayor especialización relativa del empleo en la industria cárnica y láctea, con lo que se observa una continuidad en la cadena.

San Ramón, Sarandí Grande, Juan Lacaze, Nueva Helvecia, Florida y San José reflejan una mayor especialización productiva relativa en la cadena láctea (fases primaria e industrial), aunque en el caso de Juan Lacaze existe además una combinación con la cadena forestal, y en Nueva Helvecia se destaca también la preponderancia de servicios logísticos.
Lascano, Río Branco, Melo, Artigas y Treinta y Tres registran una mayor especialización relativa en sistemas que combinan el cultivo de arroz y la ganadería (también los servicios en predio son relevantes en estas localidades, y la producción de soja, forestal y el sector logístico también son importantes en algunos de estos casos). En el caso particular de Río Branco cobra importancia relativa en la ocupación el sector comercial no asociado a las CPA estudiadas.

Trinidad, Durazno, Sarandí del Yi y Paso de los Toros registran un perfil asociado a la ganadería vacuna, servicios en predio y sector logístico. El sector forestal también resulta relevante en la generación de empleo en las ciudades del departamento de Durazno. En tanto, Dolores y Fray Bentos registran un perfil productivo fuertemente asociado a la logística, dinamizado en ambos casos por la agricultura extensiva y el sector forestal.

Cardona, Florencio Sánchez, Nueva Palmira y Carmelo reflejan una fuerte especialización productiva relativa en servicios logísticos, lo cual tiene su lógica si se considera la cercanía de uno de los más importantes puertos marítimos de cargas del país en Nueva Palmira. Estas tres ciudades muestran un marcado perfil agrícola en la ocupación, aunque la participación del empleo en el sector forestal no es despreciable. En los casos de Cardona, Florencio Sánchez y Carmelo destacan la participación en la industria láctea. En el último caso, también sobresalen los servicios vinculados a la actividad turística. 
Por su parte, Young presenta una vocación productiva dedicada al sector agropecuario y agroindustrial, con especialización en diversos subrubros de las CPA y se constata, junto a las ciudades de Mercedes y Paysandú (que tienen un perfil un tanto más diversificado) una combinación de sistemas productivos mixtos (agrícolaganaderos), con una fuerte provisión de servicios en predio y de logística.
Por otra parte, resulta interesante analizar aquellas CIU que reflejan una preponderancia de otras actividades primarias y agroindustrias no consideradas dentro del estudio. Es el caso por ejemplo de Bella Unión, donde predomina el cultivo de caña de azúcar y la horticultura; Salto, especializado en la producción de cítricos y en horticultura, y Paysandú, con una fuerte presencia de las industrias laneras y del cuero y de la actividad citrícola.

Figura 4. Coeficiente de especialización productiva aproximada por empleo para ciudades intermedias de Uruguay por actividad económica ${ }^{4}$

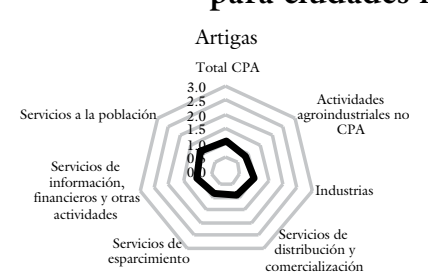

Santa Lucía
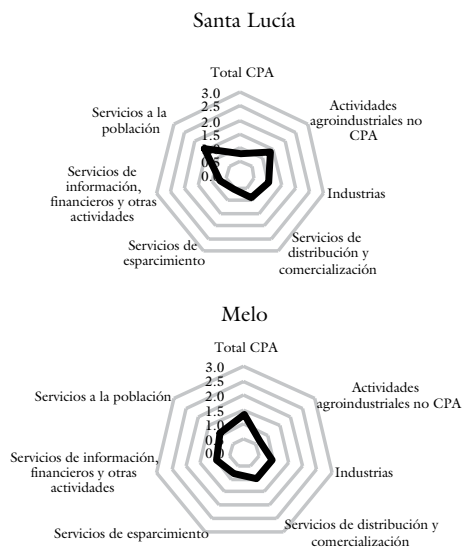
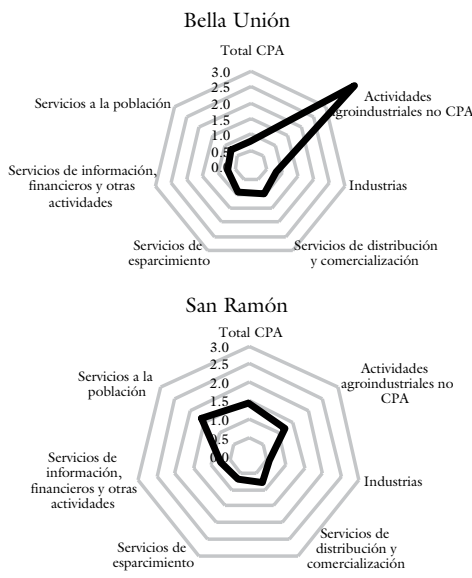

Río Branco

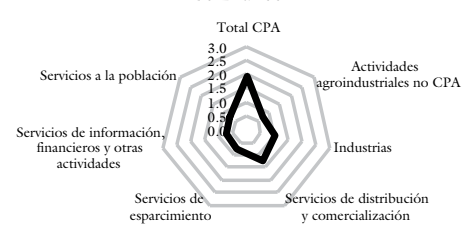

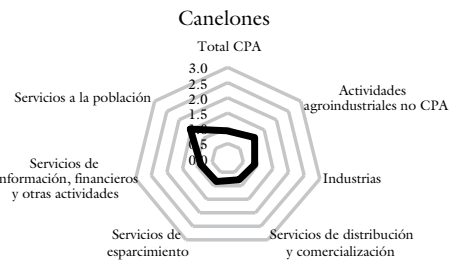

Tala

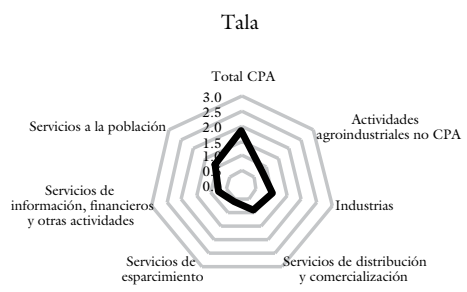

Colonia del Sacramento

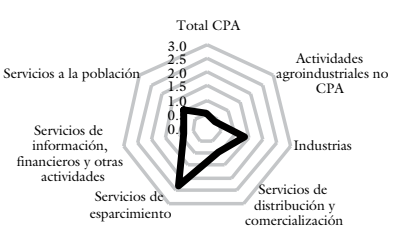

${ }^{4}$ Las actividades económicas se agruparon en las siguientes categorias (en sentido horario): Total CPA; Actividades agroindustriales no CPA; Industrias; Servicios de distribución y comercialización; Servicios de esparcimiento; Servicios de información, financierosy otras actividades; Servicios a la población. territarios 43-Especial

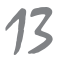




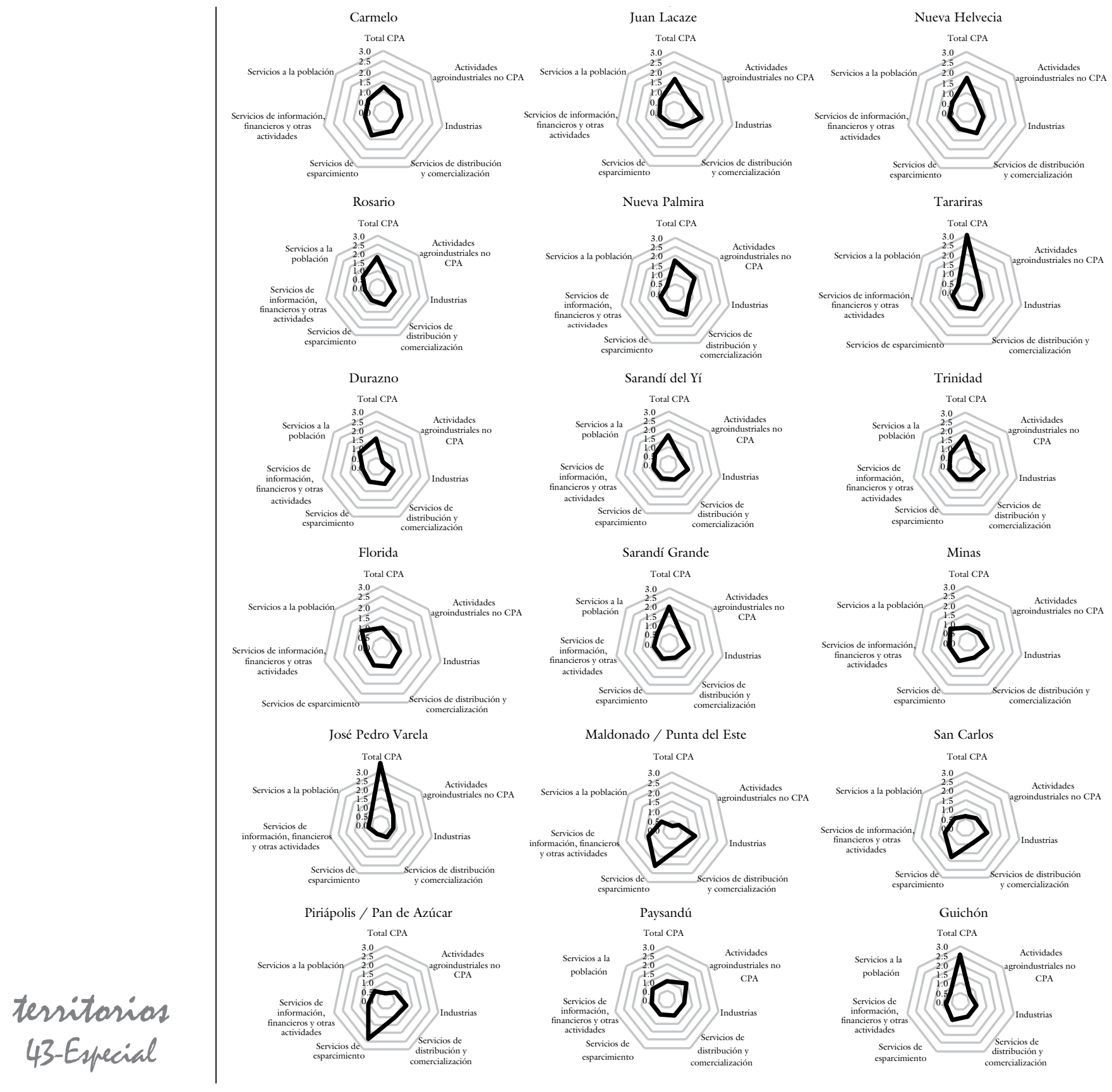




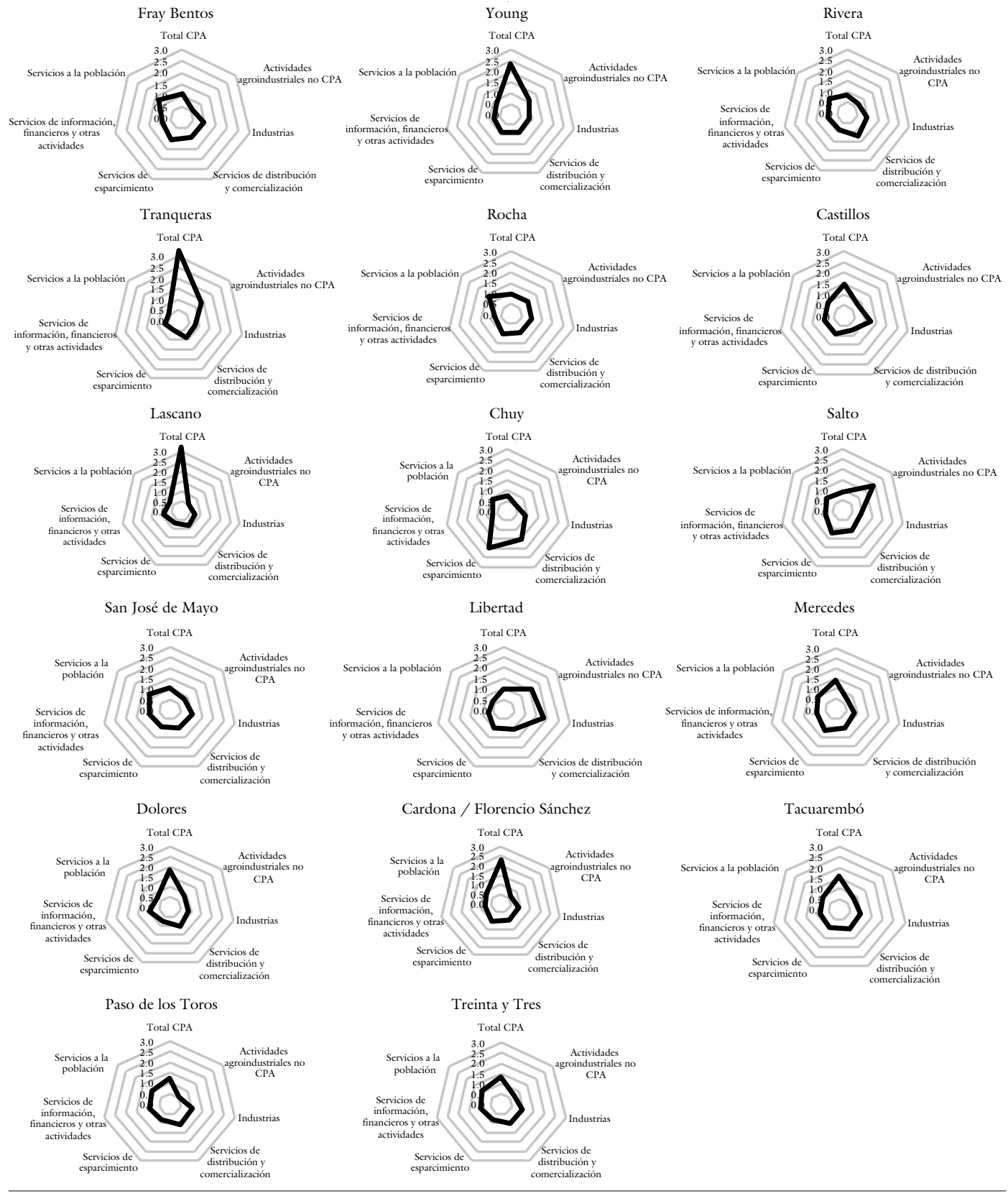

Fuente: Elaboración propia con base en ECH 2013 a 2017 del INE.

CADENAS AGROINDUSTRIALES Y VOCACIÓN PRODUCTIVA DE CIUDADES INTERMEDIAS DE URUGUAY tersitarias 43-Especial 


\section{Una propuesta de categorización de las CIU de acuerdo con su diversificación y especialización productiva}

Según Henderson (1997) los patrones de especialización de las ciudades responden, en parte, a las interacciones económicas observadas en un determinado territorio y en torno a un determinado sector de actividad (economías de localización), mientras que los patrones de diversificación de los centros urbanos están asociados con la interacción registrada entre distintos sectores de actividad a la vez (economías de urbanización). Duranton y Puga (1999) destacan que las ciudades más grandes tienden a ser más diversificadas que las de menor tamaño relativo, aunque a veces este fenómeno no es tan fácilmente observable, ya que todos los centros urbanos suelen registrar alguna proporción de empleo local asociado a actividades económicas denominadas no transables (típicamente: servicios provistos por el Estado como salud, educación, defensa, administración pública y otros servicios sociales).

A partir del análisis conjunto de los distintos indicadores de especialización y diversificación construidos se propone una categorización general de las CIU, con base en su perfil productivo.

- Centros urbanos con fuerte presencia del Estado: aquellos principalmente dedicados a la administración pública y defensa, salud, servicios sociales y enseñanza. Según el mVotma y el ITU (2016), con base en un análisis del sistema urbano nacional a partir de la movilidad de pasajeros, existen algunas CIU que ofician de referente a localidades circundantes en materia de servicios generales. En algunos casos, son las capitales departamentales las que ocupan este rol, aunque no siempre. En este grupo se destacan Artigas, Canelones, San Ramón, Durazno, Florida, Minas (que también presenta cierto predominio de construcción y de actividades turísticas y de recreación), Fray Bentos, Rocha, Paso de los Toros y Treinta y Tres (en este último caso también se constata cierta relevancia de las CPA estudiadas).

- Ciudades con manejo intensivo de recursos naturales y transformadores de materias primas e insumos con destinos diversos (alimentación y vestimenta, entre otros): incluye tanto las fuertemente dependientes de la dotación de recursos en el territorio, así como la industria trasformadora no dependiente de la existencia del capital natural en el territorio. Por un lado, Tranqueras, José Pedro Varela, Lascano, Tarariras, Cardona y Florencio Sánchez, Guichón, Young, Sarandí Grande, Tala y Dolores se destacan por una marcada especialización en actividades definidas dentro de las CPA. En menor medida Rosario, Nueva Helvecia, Río Branco (también con cierta predominancia de la actividad 
comercial, debido a su cercanía con la frontera con Brasil), Juan Lacaze (con otras industrias asociadas a la actividad agropecuaria no comprendidas en las CPA) y Tacuarembó (con cierta relevancia de los servicios asociados al Estado). Por su parte, Bella Unión y Salto muestran un marcado perfil productivo orientado a actividades primarias no comprendidas dentro de las CPA. En lo que se refiere a la especialización en industrias manufactureras, Paysandú (con una estructura productiva bastante diversificada) refleja una predominancia del empleo asociado a industrias procesadoras de materias primas agropecuarias no definidas dentro de las CPA, mientras Libertad registra un predominio de la ocupación en industrias no vinculada a las CPA (por ejemplo: autopartes e industria química).

- Con vocación en servicios de apoyo transversales: centros urbanos con una mayor relevancia del empleo vinculado a construcción, comercio, transporte y almacenamiento. Es el caso de Nueva Palmira que, por su cercanía a uno de los puertos graneleros más importantes del país, refleja una fuerte especialización en servicios logísticos, y de Sarandí del Yí y Rivera (debido a la actividad comercial asociada a la frontera).

- Con vocación turística y en servicios culturales: CIU dedicadas principalmente a servicios de alojamiento y comida, arte y recreación. Dentro de este grupo se identifica a Colonia del Sacramento y Carmelo en el litoral oeste, y a Maldonado, San Carlos y Piriápolis/Pan de Azúcar, en el este. En tanto, Chuy se destaca en la frontera con Brasil.

- Centros proveedores de servicios empresariales: asociados a servicios profesionales, técnicos e inmobiliarios y a la actividad financiera y de seguros. No se detectaron en el presente estudio CIU especializadas en servicios empresariales. Esto podría explicarse porque las oficinas centrales de bancos, casas financieras, compañías de seguros y firmas proveedoras de servicios profesionales se encuentran ubicadas en Montevideo, por lo que muy probablemente el empleo asociado a dichas actividades tenga un mayor protagonismo relativo en la capital del país.

\section{Reflexiones finales}

A partir de las actividades desarrolladas por los ocupados, se caracterizó la especialización de las ciudades de más de 5000 habitantes del interior del país. Esto permitió encontrar patrones de diversificación productiva o concentración de actividades económicas, en particular en las CPA estudiadas.

De las 44 localidades analizadas, 32 presentaron un CEE superior al promedio de la economía, lo que indica que en gran territorias 43-Especial 


\section{territorias} 43-Especial medida las CIU dependen de manera significativa de estas actividades. A su vez, 24 de las 44 localidades intermedias analizadas registran un CEE máximo y superior a la unidad en las CPA exploradas.

Del análisis de los indicadores construidos con base en el empleo, pareciera que tanto el patrón de diversificación como el de especialización de las CIU estaría vinculado tanto a la dotación de recursos naturales disponibles a nivel del territorio (lo que genera una cierta dependencia de este) como a la "vocación" productiva histórica (se constatan ciudades con especialización predominantemente forestal, agrícola, lechera, arrocera e incluso turística). Al respecto, es posible identificar ciudades con especialización predominantemente forestal (como Guichón y Tacuarembó), agrícola (como ocurre con las localidades del litoral oeste del país), lechera (según se observa en San Ramón, Sarandí Grande, Juan Lacaze, Nueva Helvecia, Florida y San José), arrocera (como José Pedro Varela, Lascano, Río Branco, Melo, Artigas y Treinta y Tres) e incluso turística (en particular en las ciudades de la costa este).

Es de destacar, además, la interacción sinérgica que se genera entre las actividades visualizadas en algunas localidades; por ejemplo, de José Pedro Varela y Tranqueras, clasificadas como las localidades con mayor especialización productiva en las CPA analizadas respecto al promedio nacional, donde se observa la presencia de sistemas pecuario-arroceros y silvo-pastoriles, respectivamente. En tanto, una interacción similar se observa en Cardona, Florencio Sánchez, Nueva Palmira y Carmelo, donde la preponderancia de la actividad agrícola y la cercanía de las dos plantas procesadoras de pasta de celulosa del país han inducido también a una mayor especialización relativa en materia de servicios logísticos.

Asimismo, el tamaño de las CIU en términos de población también pareciera tener su incidencia en su perfil productivo, se evidencia el aprovechamiento de las economías de urbanización: aquellas ciudades de mayor tamaño son más diversificadas que las de menor tamaño relativo. Así, es de destacar la mayor diversificación relativa que presentan las ciudades capitales departamentales, donde la presencia de los servicios del Estado se combina con otras actividades vinculadas a su vocación histórica.

En términos generales, es posible identificar ciudades con fuerte presencia de la administración pública y otros servicios asociados al Estado (salud, educación, servicios sociales); con fuerte vocación productiva asociada al manejo intensivo de recursos naturales o a la transformación de materias primas e insumos; con vocación en la provisión de servicios transversales (comercio, construcción, transporte y almacenamiento), y con dedicación a los servicios turísticos y la oferta de bienes culturales y recreativos. En cambio, no es posible detectar claramente la presencia de centros urbanos especializados 
en la provisión de servicios empresariales (bancarios, financieros, de seguros, profesionales y técnicos), lo cual estaría explicado por la centralidad histórica que posee la capital del país en lo referente a la localización de las principales compañías del mencionado rubro.

Estudios futuros deberían incorporar una mayor cantidad de dimensiones adicionales al empleo para evaluar el efecto distrito o los sistemas productivos locales (por ejemplo, incorporando al análisis la estructura de empresas en las ciudades). A su vez, el análisis podría ampliarse a otras áreas como agrupamientos de zonas rurales e incorporar otras dimensiones de la caracterización de los ocupados.

\section{Referencias}

Climent, E. (1997). Sistemas productivos locales y distritos industriales, el caso de España. BAGE. Boletín de la Asociación de Geógrafos Españoles, (24), 91-106. https://dialnet.unirioja.es/ servlet/articulo? codigo $=1318606$

Duranton, G., \& Puga, D. (1999). Diversity and specialisation in cities: Why, where and when does it matter? Research Papers in Environmental and Spatial Analysis, (56), 1-45. http://citeseerx. ist.psu.edu/viewdoc/download?doi=1 $0.1 .1 .319 .7903 \&$ rep $=$ repl $\&$ type $=$ pdf Goinheix, S., Rodríguez Miranda, A., Troncoso, C., Parada, C., \& Gariazzo, F. (2014). Mapeo de capacidades territoriales y especialización productiva. Oportunidades de intervención para el desarrollo local con inclusión. http://www.iecon.ccee. edu.uy/mapeo-de-capacidades-territoriales-y-desarrollo-productivo/ publicacion/384/es/

Goyeneche, J. J., Perera, M., \& Zoppolo, G. (2011). Informes del sistema de información social para la gestión local. Documento de Trabajo 21. Oficina de Planeamiento y Presupuesto.

Henderson, J. V. (1997) Externalities and industrial development. Journal of Urban Economics 42(3), 449-470. https:// doi.org/10.1006/juec.1997.2036

Jaramillo, D. (2016). Patrones de Especialización y diversificación industrial en Argentina. Un análisis a nivel regional entre 1996 y 2008. (Tesis de Licenciatura, Universidad Nacional de Mar del Plata, Argentina). http://nulan.mdp. edu.ar/id/eprint/2584

Martínez, E. J., Altmann, L., \& Rodríguez Crisci, C. (2013). Incidencia de las ciudades intermedias (CIU) en la conformación del sistema urbano nacional. R. Revista de la Facultad de Arquitectura, Diseño y Urbanismo, (17). http:// www.revista.edu.uy/11/2013/10/02/ incidencia-de-las-ciudades-intermedias-ciu-en-la-conformacion-del-sistema-urbano-nacional/

Naclario, A. (2010). Sistemas Productivos Locales. Politicas Publicas y Desarrollo Económico. Proyecto Programa Naciones Unidas para el Desarrollo - PNUD ARG/05/024. https://www. territarios 43-Especial 


$\mid \begin{gathered}\text { undp.org/content/dam/argentina/ } \\ \text { Publications/Desarrollo\%20Inclu- } \\ \text { sivo/Sistemas\%20Productivos\%20 } \\ \text { Locales.pdf } \\ \text { Rodríguez Miranda, A. (2011). Diagnóstico } \\ \text { de cohesión territorial para Uruguay. } \\ \text { Insumos para la formulación, análisisy } \\ \text { negociación de politicas locales de desa- } \\ \text { rrollo económico y social sustentablesen } \\ \text { Uruguay. http://www.agev.opp.gub. } \\ \text { uy/observatorio_docs/publico/222. } \\ \text { pdf } \\ \text { Rodríguez Miranda, A. (2013). Dispari- } \\ \text { dades territoriales en Uruguay: una } \\ \text { mirada desde la dimensión local del } \\ \text { desarrollo. Cuaderno de Economia } \\ \text { Segunda Época (2). https:/revistas. } \\ \text { ucu.edu.uy/index.php/cuadernodee- } \\ \text { conomia/article/view/406/378 }\end{gathered}$

Uruguay, Instituto Nacional de Estadística. (s.f.). Encuesta Continua de Hogares [2013-2017]. http://www.ine.gub. uy/web/guest/encuesta-continuade-hogaresl

Uruguay, Ministerio de Vivienda, Ordenamiento Territorial y Medio Ambiente (MVOTMA), Instituto de Teoría y Urbanismo (ITU). (2016). Sistema Urbano Nacional del Uruguay: una caracterización con base en la movilidad de pasajeros. https://otu.opp.gub.uy/ sites/default/files/docsBiblioteca/Sistema\%20urbano\%20nacional_UY.pdf Vázquez Barquero, A. (2005). Las nuevas fuerzas del desarrollo. Antoni Bosch Editor. 


\section{Anexo}

\section{Anexo 1. Correspondencia CIIU revisión 4 a cadenas $\mathrm{y}$ fases de las actividades CPA consideradas}

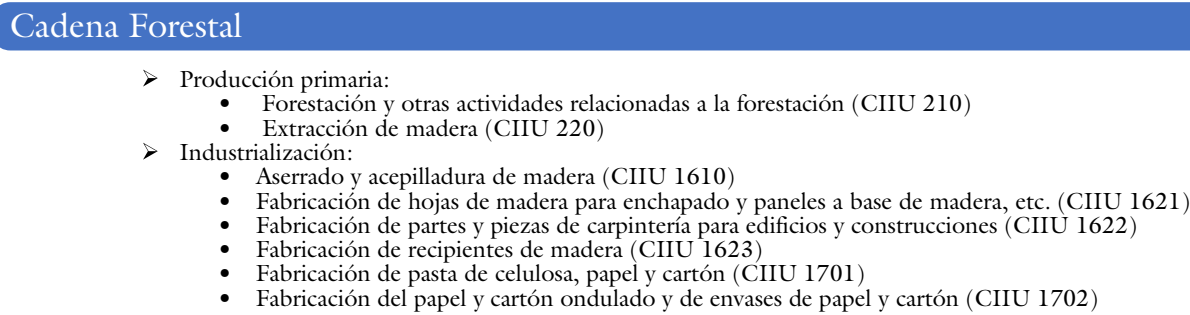

$>$ Producción primaria:

- Cultivo de arroz (CIIU 112)

$>$ Industrialización:

- Elaboración de productos de molinería (CIIU 1061). Observaciones: incluye molinos de otros cereales (trigo, harina). Sobreestimado

\section{Cadena Ganadería de carne}

$>$ Producción primaria:

- Cría de ganado vacuno y búfalos (CIIU 141). Observaciones: no es posible a priori desagregar la cría de ganado para carne y leche

$>$ Industrialización

- Matanza de ganado y otros animales (excepto aves) conservación y preparación de sus carnes (CIIU 1011)

\section{Cadena Lechería}

$>$ Producción primaria:

- Cría de ganado vacuno y búfalos (CIIU 141). Observaciones: no es posible a priori desagregar la cría de ganado para carne y leche

$>$ Industrialización

- Elaboración de productos lácteos (CIIU 1050) 
$>$ Servicios a la producción (en predio)

- Servicios de apoyo a la forestación (CIIU 240)

- Actividades de apoyo a la agricultura (CIIU 161), actividades posteriores a la cosecha (CIIU 163), procesamiento de semillas para la propagación (CIIU 164). Observaciones: no es posible desagregar lo vinculado al cultivo de soja $\mathrm{y}$ arroz

- Actividades de apoyo a la ganadería (CIIU 162). Observaciones: no es posible desagregar la cría de ganado para carne y leche

$>$ Comercialización

- Comercio al por mayor de materias primas agropecuarias y animales vivos (CIIU 4620). Observaciones: no es posible desagregar lo vinculado a las distintas cadenas

\section{Todas las CPA (transversales)}

$>$ Provisión de insumos y bienes de capital agropecuarios

- Fabricación de abonos y compuestos de nitrógeno (CIIU 2012). Observaciones: no es posible desagregar lo vinculado a las distintas cadenas

- Fabricación de pesticidas y de otros productos químicos de uso agropecuario (CIIU 2021). Observaciones: no es posible desagregar lo vinculado a las distintas cadenas

- Fabricación de maquinaria agropecuaria y forestal (CIIU 2821). Observaciones: no es posible desagregar lo vinculado a las distintas cadenas

- Fabricación de maquinaria para la elaboración de alimentos, bebidas y tabaco (CIIU 2825). Observaciones: incluye otras actividades distintas de las agropecuarias. Sobreestimado

- Comercio al por mayor de maquinaria, equipo agrícola y sus suministros (CIIU 4653). Observaciones: no es posible desagregar lo vinculado ads distintas cadenas

Logística (transporte, manipulación de cargas y almacenamiento)

- Transporte de carga por vía férrea (CIIU 4912). Observaciones: incluye otras actividades distintas de las agropecuarias. Sobreestimado

- Transporte de carga por carretera (CIIU 4923). Observaciones: incluye otras actividades distintas de las agropecuarias. Sobreestimado

- Transporte marítimo y de cabotaje de carga (CIIU 5012). Observaciones: incluye otras actividades distintas de las agropecuarias. Sobreestimado

- Transporte de carga, por vías de navegación interiores (CIIU 5022). Observaciones: incluye otras actividades distintas de las agropecuarias. Sobreestimado

- Depósito y almacenaje (CIIU 5210). Observaciones: incluye otras actividades distintas de las agropecuarias. Sobreestimado

\section{territorias 43-Especial}


Anexo 2: Coeficiente de Especialización Productiva por empleo por CIU, tamaño muestral alcanzado, proporción estimada de ocupados en CPA en el total de la localidad e intervalo de confianza al $95 \%$

\begin{tabular}{|c|c|c|c|c|}
\hline \multirow{2}{*}{ CIU } & \multirow{2}{*}{\begin{tabular}{|c|}
$\begin{array}{c}\text { Proporción } \\
\text { estimada }\end{array}$ \\
[ocupados CPA / \\
ocupados ciudad]
\end{tabular}} & \multirow{2}{*}{$\begin{array}{c}\text { Tamaño muestra } \\
\text { (5 ECHs })\end{array}$} & \multicolumn{2}{|c|}{ Intervalo de confianza al $95 \%$} \\
\hline & & & $\begin{array}{c}\text { Límite } \\
\text { inferior IC }\end{array}$ & $\begin{array}{c}\text { Límite } \\
\text { superior IC }\end{array}$ \\
\hline Artigas & 0,1076 & 3724 & 0,0976 & 0,1175 \\
\hline Bella Unión & 0,0759 & 1642 & 0,0631 & 0,0887 \\
\hline Canelones & 0,0931 & 1850 & 0,0798 & 0,1063 \\
\hline Santa Lucía & 0,0794 & 1317 & 0,0648 & 0,094 \\
\hline San Ramón & 0,1436 & 706 & 0,1177 & 0,1695 \\
\hline Tala & 0,1867 & 459 & 0,1511 & 0,2224 \\
\hline Melo & 0,1335 & 4101 & 0,1231 & 0,1439 \\
\hline Río Branco & 0,1914 & 1091 & 0,168 & 0,2147 \\
\hline Colonia del Sacramento & 0,054 & 2277 & 0,0447 & 0,0633 \\
\hline Carmelo & 0,1251 & 1637 & 0,1091 & 0,1411 \\
\hline Juan Lacaze & 0,1623 & 1080 & 0,1403 & 0,1842 \\
\hline Nueva Helvecia & 0,1744 & 980 & 0,1507 & 0,1982 \\
\hline Rosario & 0,1791 & 785 & 0,1522 & 0,2059 \\
\hline Nueva Palmira & 0,1769 & 722 & 0,149 & 0,2047 \\
\hline Tarariras & 0,304 & 481 & 0,2629 & 0,3451 \\
\hline Durazno & 0,1557 & 3054 & 0,1428 & 0,1685 \\
\hline Sarandí del Yí & 0,1658 & 605 & 0,1362 & 0,1955 \\
\hline Trinidad & 0,1668 & 2322 & 0,1517 & 0,182 \\
\hline Florida & 0,1003 & 3057 & 0,0896 & 0,1109 \\
\hline Sarandí Grande & 0,2009 & 654 & 0,1702 & 0,2316 \\
\hline Minas & 0,077 & 3253 & 0,0678 & 0,0861 \\
\hline
\end{tabular}




\begin{tabular}{|c|c|c|c|c|}
\hline \multirow{2}{*}{ CIU } & \multirow{2}{*}{$\begin{array}{c}\begin{array}{c}\text { Proporción } \\
\text { estimada }\end{array} \\
\text { [ocupados CPA / } \\
\text { ocupados ciudad] }\end{array}$} & \multirow{2}{*}{$\begin{array}{c}\text { Tamaño muestra } \\
(5 \mathrm{ECHs})\end{array}$} & \multicolumn{2}{|c|}{ Intervalo de confianza al $95 \%$} \\
\hline & & & $\begin{array}{c}\text { Límite } \\
\text { inferior IC }\end{array}$ & $\begin{array}{c}\text { Límite } \\
\text { superior IC }\end{array}$ \\
\hline José Pedro Varela & 0,3494 & 420 & 0,3038 & 0,395 \\
\hline Maldonado/Punta del Este & 0,0225 & 11272 & 0,0198 & 0,0253 \\
\hline San Carlos & 0,0649 & 3024 & 0,0561 & 0,0737 \\
\hline Piriápolis/Pan de Azúcar & 0,04 & 1784 & 0,0309 & 0,0491 \\
\hline Paysandú & 0,1046 & 6878 & 0,0974 & 0,1119 \\
\hline Guichón & 0,259 & 461 & 0,219 & 0,299 \\
\hline Fray Bentos & 0,1096 & 2196 & 0,0965 & 0,1226 \\
\hline Young & 0,2372 & 1313 & 0,2141 & 0,2602 \\
\hline Rivera & 0,086 & 6635 & 0,0793 & 0,0928 \\
\hline Tranqueras & 0,3332 & 685 & 0,2979 & 0,3684 \\
\hline Rocha & 0,0953 & 3694 & 0,0859 & 0,1048 \\
\hline Castillos & 0,1455 & 1006 & 0,1237 & 0,1673 \\
\hline Lascano & 0,3229 & 1017 & 0,2942 & 0,3517 \\
\hline Chuy & 0,0744 & 1331 & 0,0603 & 0,0885 \\
\hline Salto & 0,0909 & 8661 & 0,0848 & 0,0969 \\
\hline San José de Mayo & 0,1073 & 3545 & 0,0971 & 0,1175 \\
\hline Libertad & 0,1005 & 1098 & 0,0827 & 0,1183 \\
\hline Mercedes & 0,1419 & 3978 & 0,1311 & 0,1528 \\
\hline Dolores & 0,1849 & 1304 & 0,1638 & 0,206 \\
\hline $\begin{array}{l}\text { Cardona/Florencio } \\
\text { Sánchez }\end{array}$ & 0,2301 & 527 & 0,1942 & 0,266 \\
\hline Tacuarembó & 0,1593 & 4663 & 0,1488 & 0,1698 \\
\hline Paso de los Toros & 0,1218 & 1286 & 0,104 & 0,1397 \\
\hline Treinta y Tres & 0,1361 & 3056 & 0,124 & 0,1483 \\
\hline
\end{tabular}

